Central European Journal of Energetic Materials, 2016, 13(2), 483-504

ISSN 1733-7178

e-ISSN 2353-1843

\title{
Kinetics of Polymorphic Transitions in Energetic Compounds
}

\author{
Nikita V. CHUKANOV*, Viktor V. ZAKHAROV, \\ Boris L. KORSUNSKIY, Aleksandr D. CHERVONNYI, \\ Svetlana A. VOZCHIKOVA \\ Institute of Problems of Chemical Physics \\ of Russian Academy of Sciences,
}

Semenov av. 1, Chernogolovka, Moscow region, 142432, Russia

*E-mail: chukanov@icp.ac.ru

\begin{abstract}
Available data on the kinetics of polymorphic transitions (PTs) in energetic compounds under isothermal conditions are summarized and discussed. It is shown that the general kinetic regularities of these processes (stepwise and continuous regimes) depend on their topotactic mode (frontal or quasihomogeneous, respectively). In reverse PTs, a nucleation stage is not observed, which is explained by the presence of nuclei of the low-temperature polymorph in the preheated sample. The influence of mechanical effects on the kinetics of PTs in molecular crystals is discussed.
\end{abstract}

Keywords: polymorphic transitions, energetic compounds, molecular crystals, kinetics, calorimetry, IR spectroscopy

\section{Introduction}

The kinetics of polymorphic transitions (PTs) in energetic solids remains a poorly studied field of science. However it is difficult to overestimate its value. In the case of molecular crystals, to which the majority of highly-energetic substances belong, the PTs occur at relatively low temperatures because these processes are not accompanied by the cleavage of strong covalent bonds. Therefore a PT often precedes processes such as burning and explosion (see e.g. [1,2]). As a result, it is not the initial substances, but products of their PTs possessing specific properties 
that are involved in these processes. It should be noted that some components of propellants and explosives are metastable polymorphs. When a high density modification is metastable under normal conditions, e.g. in the case of the $\varepsilon$-modification of 2,4,6,8,10,12-hexanitro-2,4,6,8,10,12-hexaazaisowurtzitane $(\varepsilon$-HNIW), its transition to a thermodynamically stable low-density polymorph can be an important factor in determining the stability of energetic materials during their preparation, processing and storage.

Polycrystalline substances are very diverse in their properties, and it is impossible to suggest any universal mechanism for their polymorphic transitions. The observed kinetic regularities are the result of a combination of different factors, including the nature of nucleation centers, the rate constants for the nucleation and growth stages, the role of mechanical strains etc. In recent years a number of interesting results in this area has been obtained. However experimental data, especially for kinetic aspects, remain very poor and often mutually inconsistent.

In $[3,4]$, the kinetics of the PT of various polymorphs of octahydro-1,3,5,7tetranitro-1,3,5,7-tetrazocine (HMX) into $\delta$-HMX was investigated by means of FTIR spectroscopy. All these processes follow the first-order kinetic law.

In [5], the isothermal kinetics of the PT $\beta \rightarrow \delta$ was investigated at different temperatures by means of quantitative Rietveld analysis of powder X-ray diffraction patterns. The kinetic curves were described by the Avrami equation. A one-dimensional mechanism, including random nucleation and growth, was suggested.

Contrary to these data, in [6] the kinetic curves obtained for the PT $\beta \rightarrow \delta$ in HMX, by means of second harmonic generation experiments, have sigmoid character with a pronounced induction period. The transformation is accompanied by the disintegration of crystals. It was shown that nucleation occurs at one or two centers within a crystal and is followed by the growth of the $\delta$-phase starting from these centers. The duration of the induction period can differ widely in different crystals. In the same work, the reverse transition $\delta \rightarrow \beta$ was studied. In this process the consumption of $\delta$-HMX follows the first-order kinetic law. A theoretical interpretation of these data is given in [7]. The kinetic model suggested in this work is based on the assumptions that the PT is reversible, the nucleation stage follows the first-order kinetic law and the growth stage of a new phase follows the second-order kinetic law. The integral equations for the rate obtained, based on this model, describe sigmoidal temporal dependences both for the direct and reverse PTs, which contradicts the experimental data published by the same authors in [6]. A kinetic model for the growth of a new phase constructed in [7] involves an activated transition state 
which is thermodynamically equivalent to the melt.

Two more kinetic models of the PT $\beta \rightarrow \delta$ in HMX are suggested in [8]. One of them (the detailed model) contains separate kinetic parameters for the nucleation process, including an activation energy resulting from a redistribution of defect energies, and for the movement of the resulting reaction interface within a single particle. The dependences of the PT rates on temperature were chosen in such a way that they go to zero at the transition temperature. Another model (the global model) is based on the extended Prout-Tompkins nucleation-growth formalism with an added thermodynamic inhibition term (as in the detailed model, the PT rates go to zero at the transition temperature). It was concluded that the activation energy for nucleation $(333 \mathrm{~kJ} / \mathrm{mol})$ is substantially higher than that for growth $(29.3 \mathrm{~kJ} / \mathrm{mol})$.

The concept of internal, stress-induced virtual melting for a HMX crystal embedded in a polymeric binder [9] is of particular interest. According to this concept, during nucleation, nano-sized clusters of $\beta$-HMX dissolve in a molten binder and transform diffusionally into $\delta$ phase clusters. During the interface propagation, internal stresses induced by transformation strain cause melting of the stressed $\delta$ phase much below the melting temperature and its immediate resolidification into the unstressed $\delta$ phase.

The DSC method was used for the investigation of the PT $\beta \rightarrow \delta$ in HMX [10]. In experiments carried out at heating rates from 1 to $10{ }^{\circ} \mathrm{C} / \mathrm{min}$, fine structure (numerous narrow peaks) imposed on the main DSC curve was observed, particularly at the lower heating rates. The authors supposed that this was due to uneven contact of the powdered sample with the sample pan as a result of changes in morphology and density of the crystals in the course of the PT. A similar fine structure was observed in DSC curves for the PT $\varepsilon \rightarrow \gamma$ in HNIW [11] and $\alpha \rightarrow \beta$ in 1,1-diamino-2,2-dinitroethene (FOX-7) [12], but an unambiguous explanation of the nature of these phenomena was not given in these reports. It is important to note that separate peaks in the DSC curves cannot be attributed to PTs in separate crystals: in all cases the number of peaks forming the fine structure does not exceed several tens, whereas the samples used contained many thousands of crystals.

In [13], the PT $\beta \rightarrow \delta$ in HMX was investigated in a single HMX crystal, with dimensions $250 \times 45 \mu \mathrm{m}$, at $184^{\circ} \mathrm{C}$ by means of atomic force microscopy. The formation of voids orthogonal to the $a$-axis at the crystal surface during the PT was explained to be the result of nucleation in areas due to local damage or stress fields.

The role of different factors influencing nucleation in PTs of molecular crystals remains insufficiently understood. In particular, it was found [14] that 
the presence of $5 \%$ of a polymer binder results in the acceleration of nucleation. The nature of this phenomenon is unclear.

The kinetics of PTs of other energetic compounds has been studied in less detailed than that of HMX.

In [15], solid-solid phase transitions of the HNIW (CL-20) polymorphs $\varepsilon \rightarrow \gamma$, $\alpha \rightarrow \gamma$ and $\beta \rightarrow \gamma$ were studied under isothermal conditions by transmission FTIR spectroscopy. The kinetic laws of the PTs are complex. Neither an induction period, nor self-acceleration were observed. Note that in this work the samples were subjected to mechanical exposure during the preparation of a thin uniform film sandwiched between the $\mathrm{NaCl}$ windows.

From the above compilation of literature data it is obvious that the kinetics of PTs in molecular crystals differs essentially not only from classical homogeneous kinetics, but also from kinetics of solid-phase chemical reactions. Undoubtedly, this is connected with the nature of the bonds undergoing transformation: strong covalent bonds in the case of chemical reactions and weak van der Waals and dipole-dipole interactions in the case of PTs. It is worth noting that knowledge of the main kinetic regularities of polymorphism is not limited to energetic compounds, but is of general interest [16].

In the majority of publications related to phase transitions in energetic compounds, these processes were investigated under nonisothermal conditions, mainly by means of DSC. Obviously, such an approach could not provide reliable kinetic parameters of the processes. This paper is aimed at a summary of the available data on the kinetics of PTs in energetic compounds under isothermal conditions. Research in this direction has been carried out in the Institute of Problems of Chemical Physics RAS, Chernogolovka, Russia during the last 15 years. Their results are summarized below. Different modifications of HNIW, HMX and FOX-7 were selected as the subjects. $\varepsilon$-HNIW was synthesized following the procedure described in [17]. $\alpha$-HNIW was obtained by crystallization from ethyl acetate and nitric acid. Different modifications of HMX were prepared in accordance with methods described in [18]. FOX-7 was synthesized by the method described in [19].

\section{Irreversible PT. Frontal and Quasi-homogeneous Modes}

At low pressures, the low-density $\gamma$-modification of 2,4,6,8,10,12-hexanitro$2,4,6,8,10,12$-hexaazaisowurtzitane (HNIW) is thermodynamically stable over a wide temperature range [20]. According to quantum-chemical calculations, Coulomb (dipole-dipole) interactions in the $\varepsilon$ - and $\alpha$ - modifications of HNIW 
are rather strong (Table 1). As a result, these polymorphs are metastable under normal conditions; their transitions into $\gamma$-HNIW proceed at measurable rates in the temperature interval $100-160{ }^{\circ} \mathrm{C}$.

Table 1. Van der Waals $\left(E_{\mathrm{v}}\right)$ and Coulomb $\left(E_{\mathrm{Q}}\right)$ contributions $(\mathrm{kcal} / \mathrm{mol})$ to the energies of the intermolecular interactions in HNIW polymorphs (after [21])

\begin{tabular}{|c|c|c|c|}
\hline Polymorph & $E_{\mathrm{v}}$ & $E_{\mathrm{Q}}$ & $E_{\text {Total }}$ \\
\hline$\gamma$ & -25.6 & -14.6 & -40.2 \\
\hline$\alpha\left(\mathrm{H}_{2} \mathrm{O}\right)$ & -30.0 & -8.8 & -38.8 \\
\hline$\varepsilon$ & -27.5 & -11.1 & -38.6 \\
\hline
\end{tabular}

For this reason $\varepsilon^{-}$and $\alpha$-HNIW were considered as suitable model materials for an investigation of the specific features of irreversible phase transitions in molecular crystals. At the same time, these modifications possess a number of essential differences. The PT $\varepsilon \rightarrow \gamma$ proceeds with a significant increase $\left(\Delta V / V_{0}=0.065\right)$ in crystal volume per molecule [22]. As a result, this process is accompanied by strong mechanical strain and disintegration of single crystals. In the PT $\alpha \rightarrow \gamma$, the value of $\Delta V / V_{0}$ is much lower (about 0.005). Unlike the PT $\varepsilon \rightarrow \gamma$, the transition $\alpha \rightarrow \gamma$ is not accompanied by a change of conformational state of the HNIW molecule. One more difference between $\alpha$-HNIW and $\varepsilon$-HNIW is the presence of water in the former. The $\alpha$-modification of HNIW can be considered as a pseudopolymorphic hydrate [23, 24]: water molecules are usually present in the cavities of its crystal structure, in fixed but partially vacant sites [22, 25]. However $\alpha$-HNIW can exist also in an anhydrous form [15]. The results of the investigations of irreversible PTs in HNIW under isothermal conditions were published in [15,26-31]. The main conclusions are listed below.

Microscopic observations [27-31] show that the two PT modes, frontal and quasi-homogeneous, can be realized in the HNIW polymorphs.

The frontal PT mode is characterized by a pronounced induction period $\tau$, that varies for different crystals. For $\varepsilon$-HNIW with a mean crystal size of $150 \mu \mathrm{m}$, at $150{ }^{\circ} \mathrm{C}$ the value of $\tau$ varies from 10 to $96 \mathrm{~min}$ [29]. After the completion of the induction period, formation of a phase transition wave takes place near growth defects. Just thereafter, the PT wave very rapidly (during several seconds) passes through the crystal. The commencement of the process is often observed simultaneous in several crystals. The width of the wave front varies from 5 to $20 \mu \mathrm{m}$. Ahead of the front, a zone of strained crystal can be observed in polarized light; behind the front, porous polycrystalline aggregates of $\gamma$-HNIW are formed. The latter consist of plate-like particles of size ca. $10 \times 50 \times 50 \mathrm{~nm}$. The passing 
of the PT wave through the crystal results in its partial (up to a conversion of $\sim 0.1 \%$ ) strain-induced decomposition, accompanied by the evolution of a brown gas forming bubbles in the immersion liquid. The features described lead to a conclusion about the dislocation mechanism of the PT $\varepsilon \rightarrow \gamma$ in HNIW. In molecular crystals, the dislocation core can have an energy exceeding that of the ideal crystal by $5-10 \mathrm{kcal} / \mathrm{mol}$. Consequently, the dislocation core is the area where conformational and polymorphic transformations and chemical decomposition are most probable.

Already during the induction period, growth dislocations are the places of accumulation of volatile impurities that creates local gradients of mechanical stress. During the growth stage, processes accompanied by gas emission are observed in the leading part of the wave front, whereas the PT occurs in the central part of the front where mechanical stress is maximal [29].

The frontal mode can be realized also for the PT $\alpha \rightarrow \gamma$ in HNIW with crystal sizes exceeding a critical value (from $\sim 50$ to $\sim 150 \mu \mathrm{m}$, depending on the temperature) [30].

The quasi-homogeneous PT mode differs from the frontal mode in the absence of a PT wave; each single crystal of the initial phase gradually transforms into a single crystal of another modification. This mechanism is realized e.g. in the process $\alpha \rightarrow \gamma$ in HNIW with crystal sizes below $\sim 100 \mu \mathrm{m}$ [30]. The kinetic curves of quasi-homogeneous PTs are smooth. In both frontal and quasihomogeneous modes the induction period is reduced with increasing temperature and with a reduction in crystal size; in some cases it was not observed [29, 31].

One of the most interesting specific features of frontal-mode PTs, is the presence in a polycrystalline sample of several groups of crystals, each of which is characterized by a certain value of the induction period. In other words, in a sample containing many (say $10^{4}$ to $10^{5}$ ) crystals, there is a small number of groups of crystals in each of which the PT occurs almost simultaneously, whereas the $\tau$ values for the different groups can differ markedly. As a result, the $\tau$ distribution density is discrete, and unlike quasi-homogeneous PTs (Figure 1a), the process proceeds in a stepwise mode (Figures 1b, 2). 


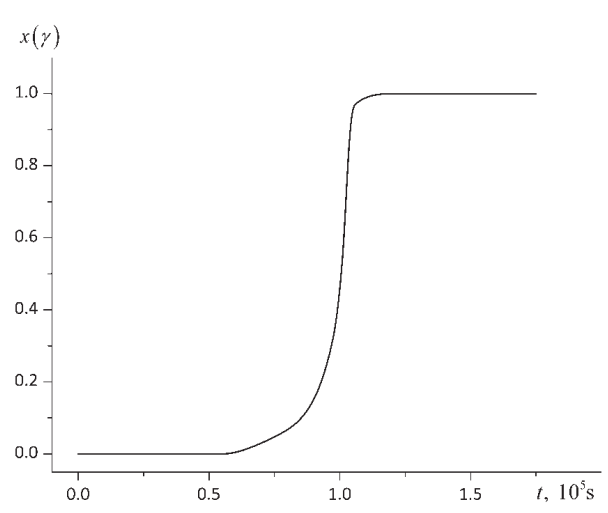

a

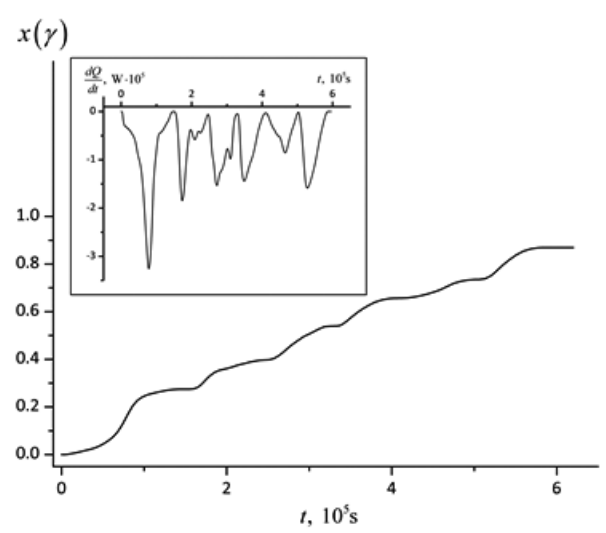

b

Figure 1. Kinetic curves of the PT $\alpha \rightarrow \gamma$ in HNIW (a) for a sample with mean crystal size $40 \mu \mathrm{m}$ at $124{ }^{\circ} \mathrm{C}$ (quasi-homogeneous PT mode), and (b) for a sample with mean crystal size $150 \mu \mathrm{m}$ at $114{ }^{\circ} \mathrm{C}$.

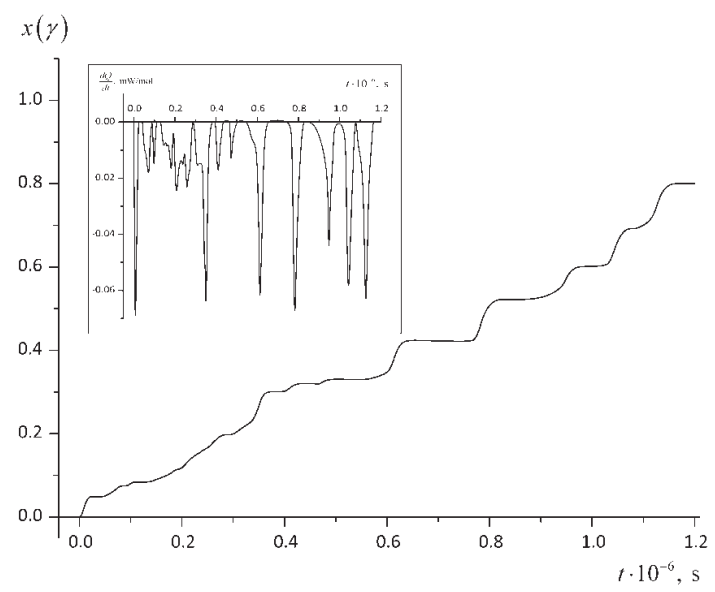

Figure 2. Integral and differential kinetic curves for the PT $\varepsilon \rightarrow \gamma$ in a HNIW sample with mean crystal size $30 \mu \mathrm{m}$ at $124.4{ }^{\circ} \mathrm{C}$ (frontal PT mode). $x(\gamma)$ is the fraction of $\gamma$-HNIW.

The stepwise PT kinetics is a rather regular phenomenon. In particular, under nonisothermal conditions it appears as multiple minima in the DSC curves of some samples of HNIW [11], HMX [10] and FOX-7 [12, 32]. As noted in [12], the "reaction profile [...] is actually a superposition of nucleation events for individual crystallites. At low heating rates, the interfacial growth of the 
phase transition is fast relative to the nucleation probability, so the features are well resolved". Under isothermal conditions this phenomenon was regularly observed by us for the PTs $\alpha \rightarrow \gamma$ and $\varepsilon \rightarrow \gamma$ in HNIW [18, 27-31] and $\beta \rightarrow \delta$ in $\operatorname{HMX}[18,33]$. The number of steps in the kinetic curves decreases with increase in temperature (Figure 3 ).

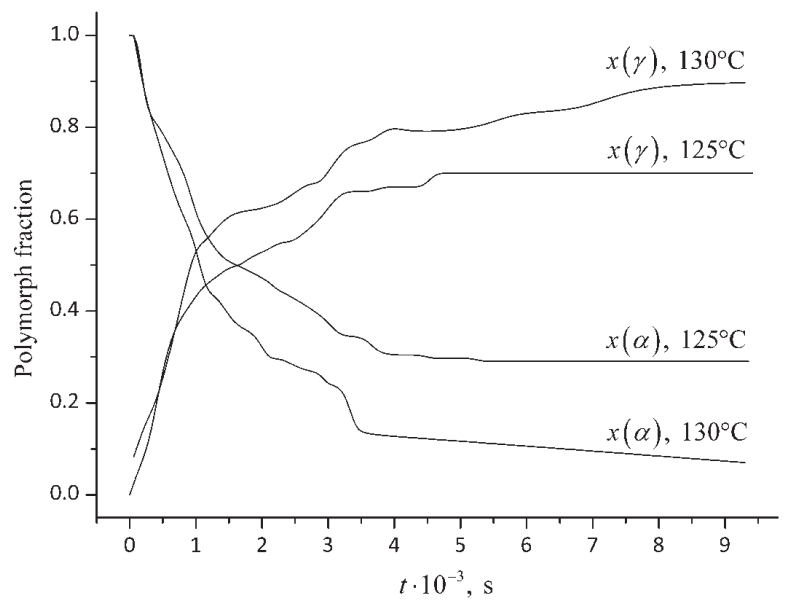

Figure 3. Kinetic curves for the PT $\alpha \rightarrow \gamma$ in HNIW with mean crystal size of $150 \mu \mathrm{m}$. The accumulation of the final phase and the consumption of the initial phase at 125 and $130^{\circ} \mathrm{C}$ have been followed by means of powder X-ray diffraction. After [31].

The discrete distribution over the induction times, resulting in the stepwise PT kinetic law, was explained by the presence of several kinds of nucleation centers in the crystal, each of which is characterized by a particular time of critical nucleus formation at a given temperature [18]. In the frontal-mode PT processes, the duration of the induction period $\tau$, in which the nucleation occurs, ranges from several seconds to several days, depending on the temperature and the kind of nucleation center. Apparently, this fact reflects the energy distinction of defects which are potential nucleation centers $[6,8]$.

This assumption is indirectly confirmed by data on the influence of crystal sizes on the kinetics of PTs. As follows from [35], the stability of $\beta$-HMX in the PT $\beta \rightarrow \delta$ is reduced with an increase in crystal size. A similar regularity was noted in [34]: a coarse sample showed phase conversion from the $\beta$ - to the $\delta$-phase, starting during the first 10 minutes at $165^{\circ} \mathrm{C}$. It required up to $4 \mathrm{~h}$ for completion. This situation is quite different for a fine sample; at $165^{\circ} \mathrm{C}$, no 
conversion was observed for the duration of the experiment. Only at $170{ }^{\circ} \mathrm{C}$, does the $\delta$-phase emerge after 90 minutes and continues to develop with time. A possible explanation of this phenomenon is that the probability of nucleation (which is the limiting stage in a frontal-mode PT) in a large crystal is greater than in a small one.

In a general case, the distribution density for the induction times is a superposition of continuous and discrete functions, which reflects differences in the nature of nucleation centers present in different crystals. In the cases of mixed-mode kinetics, the contributions of continuous and stepwise processes can be determined by means of Fourier analysis [18].

The sum of the fractions of the initial and the final phases measured for the PT $\alpha \rightarrow \gamma$ in HNIW is always equal to 1 (see e.g. Figure 3 ). However at intermediate stages of the PT $\varepsilon \rightarrow \gamma$ in HNIW, this sum, determined by means of powder X-ray diffraction, varies in the range from 0.7 to 0.9 [31]. A quantitative, full-profile analysis of the IR spectra showed that in the latter case no intermediate phases (neither amorphous, nor crystalline) are formed, and it was concluded that the lowering of the intensities of X-ray diffraction peaks is due to the formation of intermediate highly dispersed $\gamma$-HNIW [31]. This conclusion is in agreement both with our electron microscope observations [29] and with the model [7] which involves an activated transition state.

\section{Reversible PTs}

Unlike irreversible PTs, in which transformations of metastable polymorphs occur far from the border of the area of their thermodynamic stability, reversible PTs can be realized (as both direct and reverse processes) close to this border. This is possible in cases where the effective activation energy for the transformation is not too high.

The effective activation energy $E_{\mathrm{a}}$ for the initial stage of the irreversible PT $\varepsilon \rightarrow \gamma$ in HNIW at normal pressure, in the temperature interval from 143 to $161.5{ }^{\circ} \mathrm{C}$, is rather high (from $210 \mathrm{~kJ} / \mathrm{mol}$ [14] to $227 \mathrm{~kJ} / \mathrm{mol}$ [29]). The apparent activation energy increased to a maximum of about $400 \mathrm{~kJ} / \mathrm{mol}$ at $40 \%$ conversion [14]. Taking into account that the induction period and, consequently, the nucleation stage were not observed, these values should be attributed to the growth stage.

In contrast to this, the PT $\alpha \rightarrow \beta$ in FOX-7, in the temperature interval from 100.0 to $111.8^{\circ} \mathrm{C}$, is characterized by $E_{\mathrm{a}}=110 \mathrm{~kJ} / \mathrm{mol}$ [34], and is reversible.

Isothermal kinetics of the reversible PT $\beta \rightarrow \delta$ in HMX was described by 
the equation for autocatalysis, in which the order of the reaction on the catalyst increases from 1 to 2 with an increase in temperature, and the maximum rate of the reaction obeys the Arrhenius equation, with $E_{\mathrm{a}}=431 \mathrm{~kJ} / \mathrm{mol}$ [33]. However the apparent activation energy determined for the growth stage of this process [8] is very low $(29.3 \mathrm{~kJ} / \mathrm{mol})$.

In [33], several repetitive cycles of $\beta \rightarrow \delta \rightarrow \beta$ were realized in HMX. The corresponding kinetic curves of the PT $\beta \rightarrow \delta$ are given in Figure 4 . The process is accompanied by the destruction of crystals. One of the most interesting conclusions that can be made, based on these experiments, is that a reduction in the induction period and an increase in the rate of the PT $\beta \rightarrow \delta$ occurs with the increase in cycle number. Consequently, in direct PT processes, i.e. processes proceeding due to an increase in temperature above the PT point, both internal (developing as a result of the PT wave propagation), and external mechanical influence (e.g. grinding) leads to a shortening of the induction period or even to its disappearance $[14,18,28,29]$.

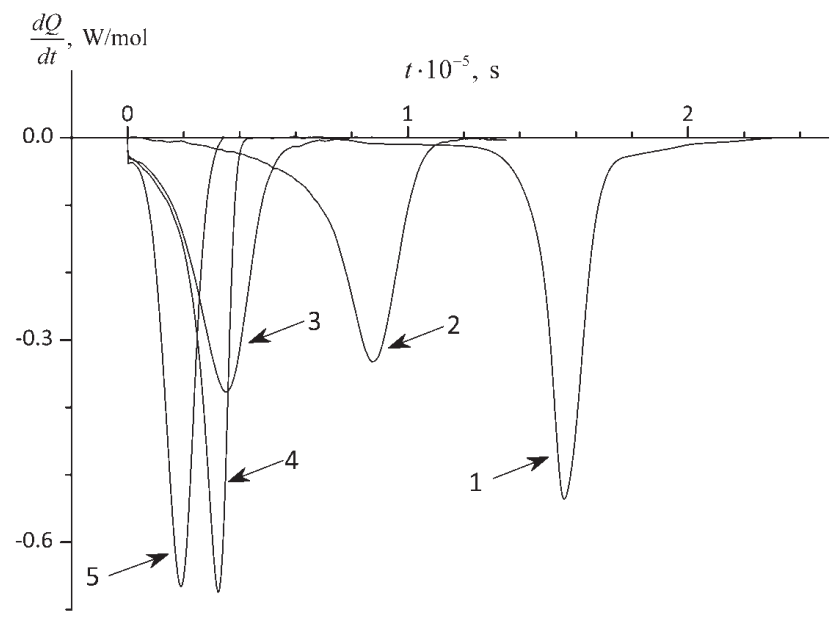

Figure 4. The differential kinetic curves of heat absorption in direct $\mathrm{PT}(\beta \rightarrow \delta)$ during several cycles $\beta \rightarrow \delta \rightarrow \beta$ in HMX with mean crystal size $20 \mu \mathrm{m}$ at $161.0{ }^{\circ} \mathrm{C}$. The figures by the curves correspond to the number of the PT cycles.

A similar trend was observed by us in repetitive PT cycles of $\alpha \rightarrow \beta \rightarrow \alpha$ in FOX-7 under non-isothermal conditions: the endothermic peak on the DSC curve in the first cycle is shifted towards higher temperatures as compared to subsequent cycles (Figure 5). 


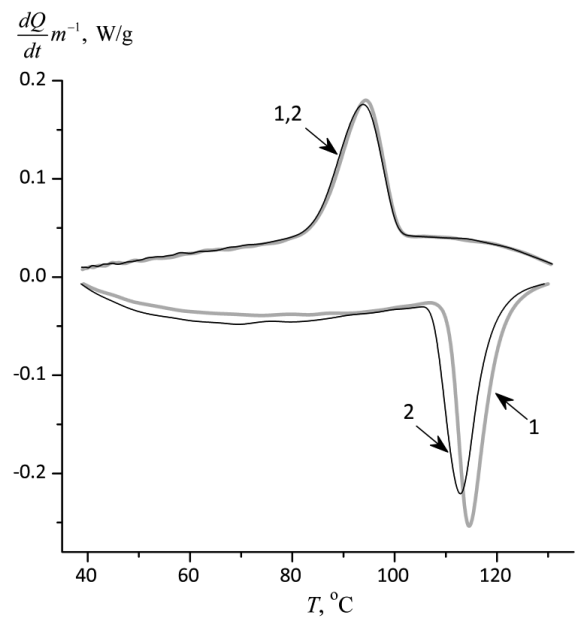

Figure 5. DSC curves for two PT cycles $\alpha \rightarrow \beta \rightarrow \alpha$ in FOX-7 with crystal sizes from 30 to $150 \mu \mathrm{m}$.

The reduction of the induction period in direct PT processes is observed also with increasing temperature [33, 34, see Figures 6, 7].

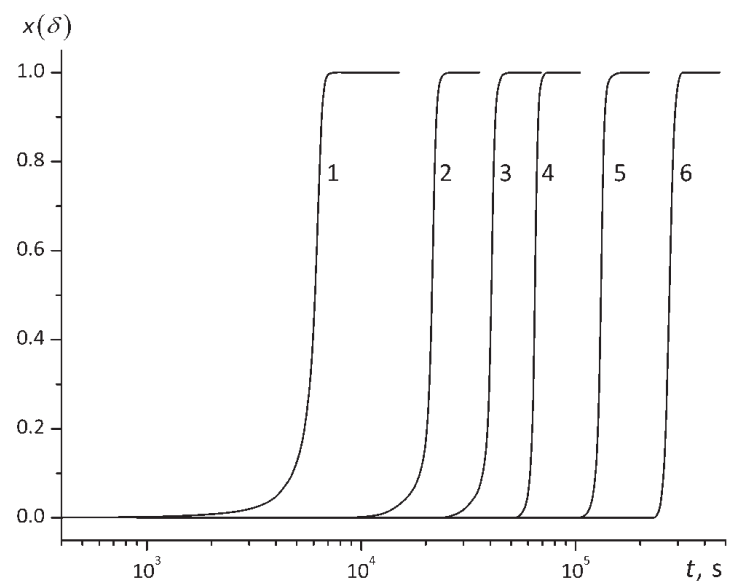

Figure 6. Kinetic curves of the PT $\beta \rightarrow \delta$ in HMX at different temperatures $\left({ }^{\circ} \mathrm{C}\right): \mathbf{1}-169.0,2-167.0,3-165.4,4-164.0,5-162.0,6-159.4$. $x(\delta)$ is the fraction of $\delta$-HMX. 


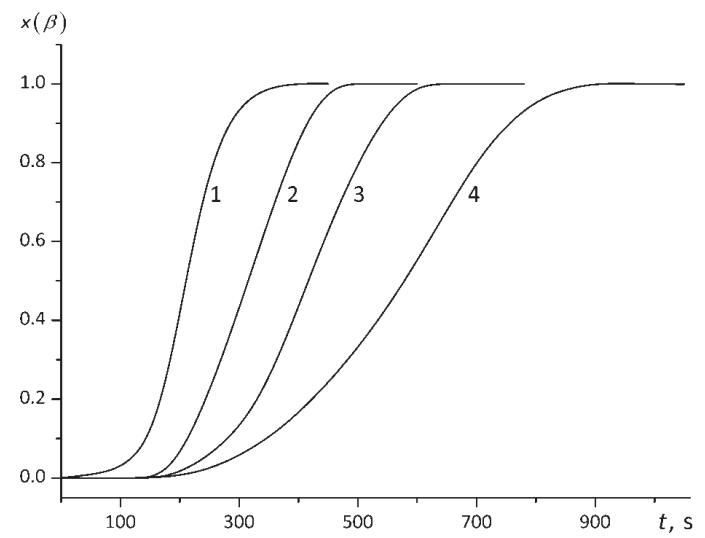

Figure 7. Isothermal kinetic curves of the PT $\alpha \rightarrow \beta$ in FOX-7 at different temperatures $\left({ }^{\circ} \mathrm{C}\right): \mathbf{1}-111.8, \mathbf{2}-108.4, \mathbf{3}-104.9, \mathbf{4}-100.0$. The kinetics follows the first-order autocatalysis law $\mathrm{d} \alpha / \mathrm{d} t=k(1-\alpha)$ $(A+\alpha)$, where $k$ is the rate constant, $\alpha$ is conversion, and $A$ ("seed concentration") is a constant [34]. $x(\beta)$ is the fraction of $\beta$-FOX-7.

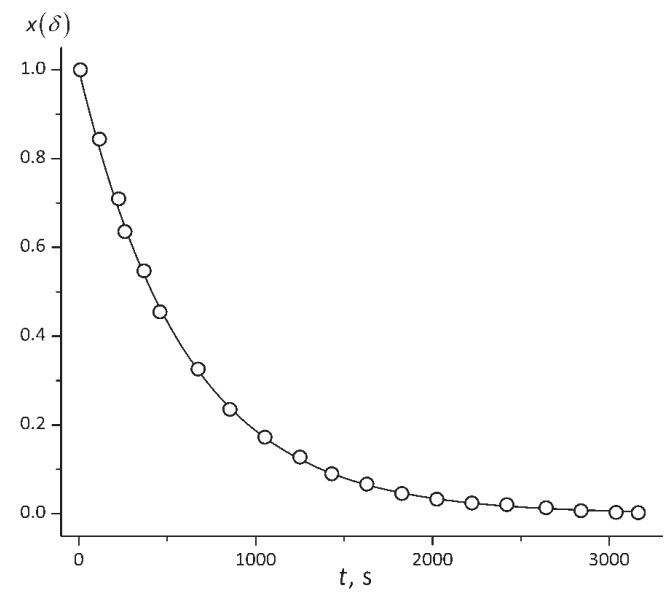

Figure 8. Kinetic curve of the PT $\delta \rightarrow \beta$ in HMX at $121{ }^{\circ} \mathrm{C}$. The points correspond to experimental data; the solid line represents the approximation in accordance with the first-order kinetic law. $x(\delta)$ is the fraction of $\delta$-HMX.

In reverse PTs proceeding due to a decrease in temperature of a preheated sample to below the PT point, both the induction period and self-acceleration are not observed. For example, the kinetics of PT $\delta \rightarrow \beta$ in HMX, obtained 
from isothermal calorimetric data [18], can be described with high accuracy by a first-order equation (Figure 8). A similar result was obtained earlier [6]. In other words, in reverse PTs the nucleation stage is not observed.

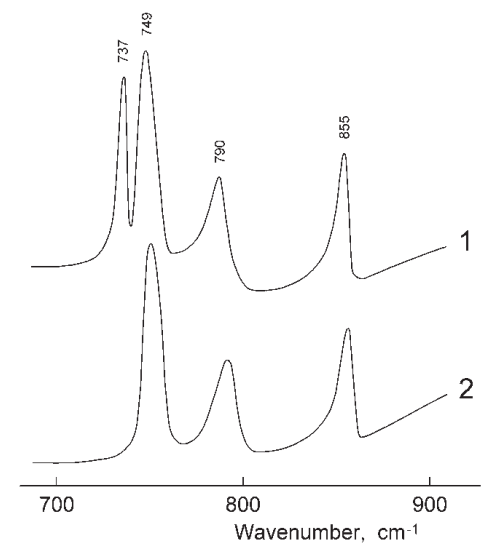

Figure 9. The IR absorption spectra of $\alpha-F O X-7$ (1) and $\beta$-FOX-7 (2) in the region of the bending vibrations of $\mathrm{NO}_{2}$ groups.

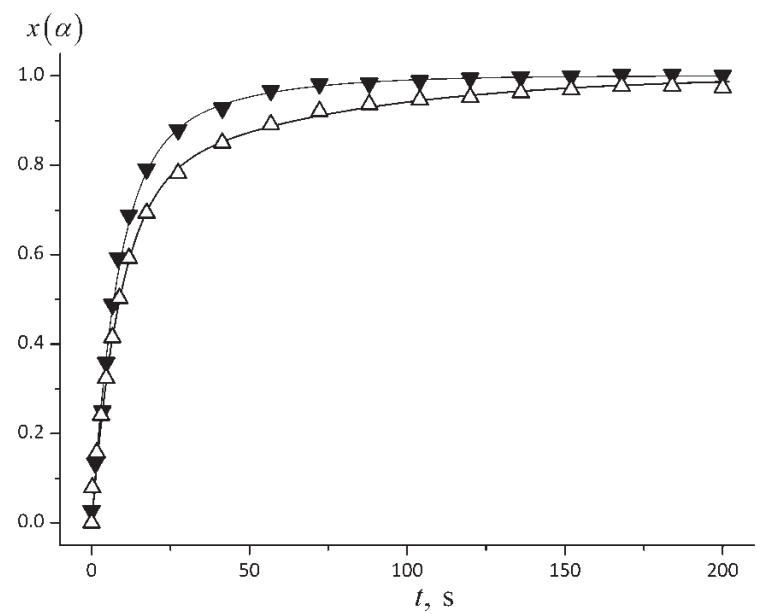

Figure 10. Kinetic curves for the PT $\beta \rightarrow \alpha$ in FOX-7 at $23{ }^{\circ} \mathrm{C}$. The symbols correspond to the experimental data, and the solid curves represent their approximation by two-exponential dependences. Black triangles correspond to a sample subjected to intense trituration in an agate mortar. $x(\alpha)$ is the fraction of $\alpha$-FOX-7. 
In [34], the kinetics of the reverse PT $\beta \rightarrow \alpha$ in FOX-7 at $23{ }^{\circ} \mathrm{C}$ was followed by the intensity of the absorption band of the bending vibrations of $\mathrm{NO}_{2}$ groups at $738 \mathrm{~cm}^{-1}$, which is present only in the IR spectrum of the $\alpha$-modification (Figure 9). The process follows the two-exponential law (Figure 10) in accordance with the equation:

$$
\alpha=1-C \exp \left(-k_{1} t\right)-(1-C) \exp \left(-k_{2} t\right)
$$

where: $\alpha$ is the conversion, $k_{1}=0.1161 \pm 0.0006 \mathrm{~s}^{-1}, k_{2}=0.0150 \pm 0.0001 \mathrm{~s}^{-1}$, $C=0.738 \pm 0.002$. In another experiment, $\alpha$-FOX-7 crystals were preliminarily vigorously triturated in an agate mortar for $1 \mathrm{~min}$. As a result, the value of $k_{2}$ doubled, whereas the other parameters of the two-exponential kinetic equation remained almost unchanged: $k_{1}=0.1266 \pm 0.0012 \mathrm{~s}^{-1}, k_{2}=0.0319 \pm 0.0007 \mathrm{~s}^{-1}$, $C=0.774 \pm 0.008$.

The causes of the differences between the kinetic laws or the above described reverse PTs, $\delta \rightarrow \beta$ in HMX and $\beta \rightarrow \alpha$ in FOX-7 (single-exponential and twoexponential, respectively), lie in the morphological features of the samples used. In the case of HMX, a fine crystalline sample characterized by a very narrow particle size distribution (average size $20 \mu \mathrm{m}$ ) was used.

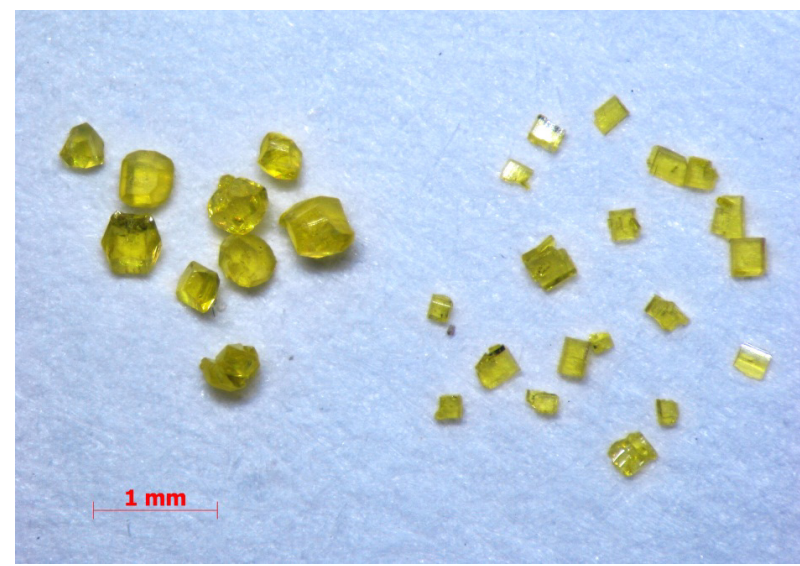

Figure 11. Two kinds of crystals present in the investigated FOX-7 sample.

In the FOX-7 sample used in [34], two kinds of crystals were present. Crystals of the first type (Figure 11, right) are parallelepiped, formed by the faces $\{100\},\{010\}$ and $\{001\}$. All dihedral angles of these crystals are close to $90^{\circ}$, which is in agreement with the angles of the orthorhombic unit cell having the parameters $a=6.939, b=6.631, c=11.349 \AA, \alpha=90^{\circ}, \beta=90.65^{\circ}, \gamma=90^{\circ}$ [37]. The 
mean sizes of these crystals were close to $250 \times 180 \times 30 \AA$, and they comprised a quarter of the sample mass. Crystals of the second type (Figure 11, left) are twinned with characteristic re-entrant angles which have isometric shapes and sizes from 0.2 to $0.8 \mathrm{~mm}$ across, the mean size being about $0.4 \mathrm{~mm}$. Note the larger sizes of the twins as compared with those of single crystals, which grew at the same time from the same solution, is a common phenomenon related to the specific features of the nucleation stage of crystallization [38].

The presence in the sample of crystals of these two types is the most probable cause of the two-exponential kinetics of the PT $\beta \rightarrow \alpha$ in FOX-7. It was shown (by means of X-ray diffraction) that the transitions between $\alpha$ - and $\beta$-modifications of FOX-7 occur in a quasi-homogeneous mode, in accordance with the mechanism single crystal $\rightarrow$ single crystal [39]. Thus it is reasonable to assume that crystals of $\beta$-FOX-7, formed on heating the different morphological varieties of the $\alpha$-polymorph, contain different kinds of structural defects. In turn, these features should influence the kinetics of the reverse transition $\beta \rightarrow \alpha$.

\section{The Role of Mechanical Effects on PTs of Molecular Crystals}

Stepwise PT kinetics is characteristic of frontal-mode PT processes in polycrystalline samples which had not been exposed to mechanical influence. Under strong mechanical treatment (e.g. crushing, pressing, or grinding in a mortar), a lot of different kinds of defects can be generated in the crystals. In addition, grinding results in the enhancement of the specific surface area, and nucleation on the surface may become dominant. As a result, the PT kinetic curves become smooth, and an induction period is not observed [14, 18, 28, 29].

Under normal conditions $\delta$-HMX transforms into a mixture of $\alpha$ - and $\beta$-modifications in a short period (from several hours to several days). Unlike $\delta$-HMX, the pseudopolymorphic hydrate $\gamma$-HMX is relatively stable. The halflife period of the PT $\gamma \rightarrow \beta$ varies from several months to several years. However this process is accelerated considerably when the crystals are ground in an agate mortar. The kinetic curves for the reverse PT $\gamma \rightarrow \beta$, obtained for a sample preliminarily triturated in an agate mortar, can be approximated by broken lines consisting of two linear segments [18].

The PT $\gamma \rightarrow \beta$ is accompanied by loss of structural water (see [40]). As was shown in [41], a solid-solid structural transformation, initiated by the release of a volatile impurity (e.g. water), follows the zero-order kinetic law when impurity mobilization is the rate determining stage of the process. Based on these data, it is reasonable to suppose that the PT $\gamma \rightarrow \beta$ is limited by water diffusion. 
In [30], the kinetics of phase transformations of $\delta$-HMX has been investigated by means of a full-profile FTIR analysis, using samples subjected to two kinds of mechanical treatment. In the first experiment, crystals of $\delta$-HMX were crushed imposing uni-axial pressure. Immediately thereafter, a rapid PT commenced. In the course of this process, $\delta$-HMX transformed to a mixture of $\alpha$ - and $\beta$-modifications (in the proportion $4.0: 1.0$ ) via the formation of intermediate $\gamma$-HMX (Figure 12). This process can be described by a formal kinetic scheme of successive transformations $\delta \rightarrow \gamma \rightarrow \alpha+\beta$, with the rate constants of the first and second stages $k_{1}$ and $k_{2}$, respectively. The analytical equations for the time dependences of the fractions of the different modifications corresponding to this scheme are:

$$
\mathrm{C}_{\delta}=\mathrm{e}^{-k 1 t} ; \mathrm{C}_{\gamma}=k_{1}\left(k_{1}-k_{2}\right)^{-1}\left(\mathrm{e}^{-k 2 t}-\mathrm{e}^{-k 1 t}\right) ; \mathrm{C}_{\alpha+\beta}=1-\mathrm{e}^{-k 1 t}-k_{1}\left(k_{1}-k_{2}\right)^{-1}\left(\mathrm{e}^{-k 2 t}-\mathrm{e}^{-k 1 t}\right) .
$$

Some deviations of the experimental data from these idealized theoretical dependences (Figure 12), including the presence of a linear segment in the curve for the consumption of $\delta$-HMX, may be connected with the inhomogeneity of the system. On the other hand, as was noted above, such behaviour can be explained by a PT mechanism in which impurity mobilization is the rate determining stage [41].

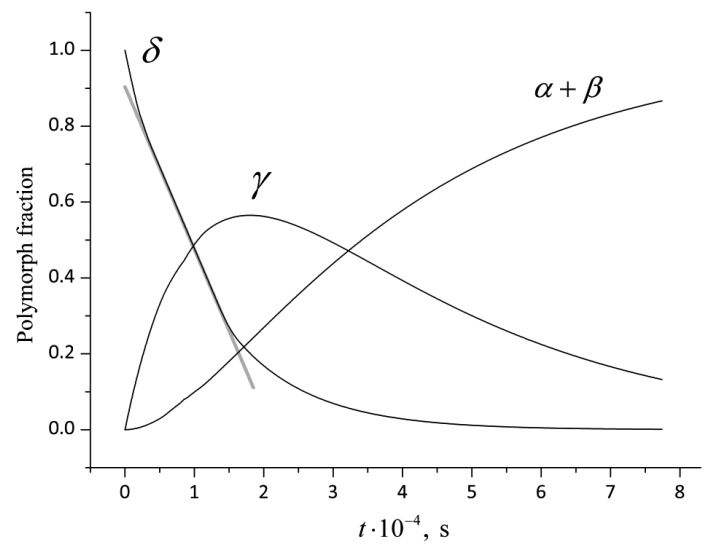

Figure 12. Experimental kinetic curves for different polymorphs in the PT of $\delta$-HMX after crushing the crystals, imposing uni-axial pressure. After [18].

In the second experiment, the $\delta$-HMX crystals were triturated in an agate mortar, i.e. subjected to shear strain. As a result, the phase composition of 
the products and the shape of the kinetic curves changed substantially as compared with those in the first experiment (Figure 13). The process can be described by the following simplified kinetic scheme: $\delta \rightarrow \alpha+\beta(\alpha: \beta \approx 3: 2)$; $\alpha \rightarrow \beta$ (a slower process). The intermediate $\gamma$-HMX is formed in insignificant amounts. In addition, shear deformation results in a significant acceleration of the consumption of $\delta$-HMX, which proceeds with self-acceleration of the initial stage. Taking into account that $\alpha-\mathrm{HMX}$ crystals obtained by crystallization from solution are stable under normal conditions, the observed transformation $\alpha \rightarrow \beta$ is an unexpected result. Hypothetically, the lowered stability of $\alpha$-HMX formed from triturated $\delta$-HMX is due to its high dispersion or high concentration of defects, which results in the acceleration of nucleation.

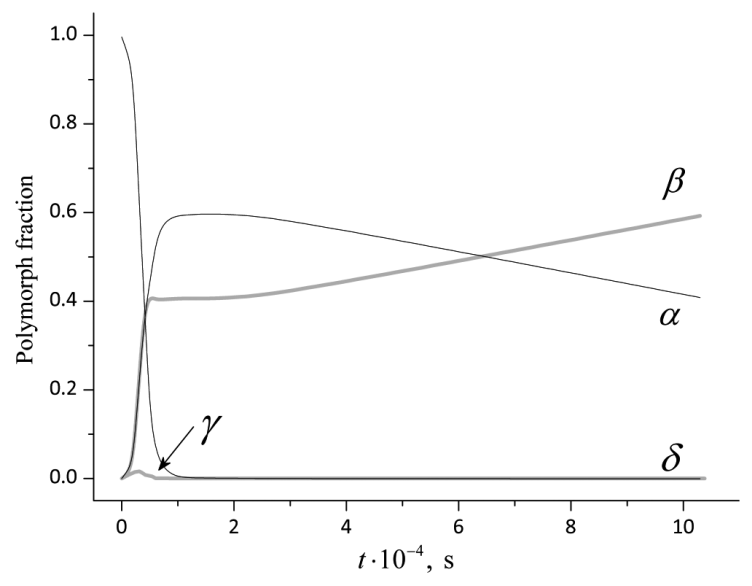

Figure 13. Kinetic curves for different polymorphs in the PTs of $\delta$-HMX after trituration in an agate mortar. After [18].

A comparison of available data on the isothermal kinetics of PTs in different polymorphs of HMX, FOX-7 and HNIW shows that these processes are characterized by a number of common specific features, but there are also some important distinctions. In all of these compounds, the PTs can occur both in frontal and in quasi-homogeneous regimes, depending on the temperature and crystal sizes. In cases when the initial phase is metastable under the conditions at which a transition occurs, the process is irreversible. The PT $\varepsilon \rightarrow \gamma$ in HNIW is an example of such a process. In some cases, the preservation of a polymorph under conditions where it is thermodynamically unstable, can be achieved at the expense of strong intermolecular interactions, as in the case of HNIW (see Table 1). Hydrogen bonds with water molecules occurring in pseudopolymorphic hydrates 
(e.g. in $\alpha$-HNIW and $\gamma$-HMX) contribute to their additional stabilization [25].

In cases where the stabilizing effect of intermolecular interactions is relatively weak, the PTs are reversible. Examples of such processes are the PTs $\beta \leftrightarrow \gamma$ in HMX and $\alpha \leftrightarrow \beta$ in FOX-7. In both cases, reverse transformations occur without an induction period and without self-acceleration.

In frontal-mode processes ( $\beta \rightarrow \delta$ in HMX, $\alpha \rightarrow \gamma$ and $\varepsilon \rightarrow \gamma$ HNIW) nucleation is the rate-determining stage, and these processes are characterized by high values of the effective activation energy of several hundred $\mathrm{kJ} / \mathrm{mol}$. The quasi-homogeneous PT $\alpha \rightarrow \beta$ in FOX-7 occurs with an activation energy of $\sim 110 \mathrm{~kJ} / \mathrm{mol}$. This value may correspond to the cleavage of several hydrogen bonds in the growth stage [34].

Unlike chemical reactions, PTs do not show a direct connection of kinetic characteristics with the chemical nature of the substance. PTs in molecular crystals are physical processes, and their specific features depend on such factors as crystal structure, density, intermolecular interactions, conformational transitions, crystal sizes, mechanical strains, the nature of defects and their concentration.

\section{Conclusions}

Based on the above data, several general conclusions can be formulated.

1. Two PT modes, frontal and quasi-homogeneous, can be realized in molecular crystals. The frontal PT mode is characterized by a pronounced induction period followed by the fast propagation of a PT wave through the crystal which results in the formation of a polycrystalline aggregate of a new phase. In quasi-homogeneous PTs, the induction period is short or is not observed; each single crystal of the initial phase transforms into a single crystal of another modification.

2. A frontal PT is often characterized by a nearly discrete distribution of induction periods in different crystals due to the presence of a small number of different kinds of nucleation centres. As a result, integral kinetic curves have a stepwise character, and differential kinetic curves (both isothermal and nonisothermal) contain multiple maxima. On the contrary, kinetic curves of quasi-homogeneous PTs are smooth.

3. In a frontal-mode PT, the $\tau$ distribution density coincides with the differential kinetic curve normalized on unit area.

4. In the case of reversible PTs, in direct processes, i.e. processes proceeding due to an increase in temperature above the PT point, both internal (developing as a result of the PT wave propagation) and external mechanical influences (e.g. 
grinding) lead to a shortening of the induction period or even to its disappearance.

5. In all known isothermal reverse PTs of energetic substances proceeding due to a decrease in temperature to below the PT point of a preheated sample, an induction period is not observed. In other words, in reverse PTs the nucleation stage is absent: nuclei of the low-temperature polymorph are initially present in the preheated sample.

6. The rate, the kinetic law and the products of PTs depend on the kind of mechanical impact on an initial sample.

\section{Acknowledgements}

This work was financially supported by the Russian Foundation for Basic Research (Grant No. 15-53-53004 NSFCa).

\section{References}

[1] Wemhoff A., Burnham A, Nichols A. III, Application of Global Kinetic Models to HMX $\beta-\delta$ Transition and Cookoff Processes, J. Phys. Chem. A, 2007, 111(9), $1575-1584$.

[2] Nedelko V., Chukanov N., Raevskii A., Korsounskii B., Larikova T., Kolesova O., Volk F., Comparative Investigation of Thermal Decomposition of Various Modifications of Hexanitrohexaazaaisowurtzitane (CL-20), Propellants Explos. Pyrotech., 2000, 25, 255-259.

[3] Brill T., Karpowitz R., Solid Phase Transition Kinetics. The Role of Intermolecular Forces in the Condensed-phase Decomposition of Octahydro-1,3,5,7-tetranitro1,3,5,7-tetrazocine, J. Phys. Chem., 1982, 86(21), 4260-4265.

[4] Karpowitz R., Brill T., Kinetic Data for Solid Phase Transitions by Fourier Transform Infrared Spectroscopy, Applied Spectroscopy, 1983, 37(1), 79-81.

[5] Xue C., Sun J., Song G., Kang B., Xia Y., Kinetics of the $\beta \rightarrow \delta$ Isothermal Phase Transition of HMX Based on Quantitative Phase Analysis Using the Rietveld Method, Explosion and Shock Waves (in Chinese), 2010, 30(2), 113-118.

[6] Smilowitz L., Henson B., Asay B., Dickson P., The $\beta-\delta$ Phase Transition in the Energetic Nitramine - Octahydro-1,3,5,7-tetranitro-1,3,5,7-tetrazocine: Kinetics, J. Chem. Phys., 2002, 117(8), 3789-3798.

[7] Henson B., Smilowitz L., Asay B., Dickson P., The $\beta-\delta$ Phase Transition in the Energetic Nitramine Octahydro-1,3,5,7-tetranitro-1,3,5,7-tetrazocine: Thermodynamics, J. Chem. Phys., 2002, 117(8), 3780-3788.

[8] Burnham A., Weese R., Weeks B., A Distributed Activation Energy Model of Thermodynamically Inhibited Nucleation and Growth Reactions and Its Application to the $\beta-\delta$ Phase Transition of HMX, J. Phys. Chem. B, 2004, 108(50), 19432-19441. 
[9] Levitas V., Henson B., Smilowitz L., Asay B., Solid-solid Phase Transformation via Virtual Melting Significantly Below the Melting Temperature, Phys. Rev. Lett., 2004, $92(23), 235702-1 / 235702-4$.

[10] Weese R, Maienschein J., Perrino C., Kinetics of the $\beta \rightarrow \delta$ Solid-solid Phase Transition of HMX, Octahydro-1,3,5,7-tetranitro-1,3,5,7-tetrazocine, Thermochim. Acta, 2003, 401(1), 1-7.

[11] Foltz M., Coon C., Garcia F., Nichols A. III, Thermal Stability of the Polymorphs of Hexanitrohexaazaisowurtzitane, Part I, Propellants Explos. Pyrotech., 1994, 19(1), 19-25.

[12] Burnham A., Weese R., Wang R., Kwok Q., Jones D., Solid-solid Phase Transition Kinetics of FOX-7, Proc. NATAS Annu. Conf., Universal City, CA, USA, 2005, $1-8$.

[13] Brandon L., Weeks R., Ruddle C., Zaug J., Cook D., Monitoring High-temperature Solid-solid Phase Transitions of HMX with Atomic Force Microscopy, Ultramicroscopy, 2002, 93, 19-23.

[14] Smilowitz L., Henson B., Greenfield M., Sas A., Asay B., Dickson P., On the Nucleation Mechanism of the $\beta-\delta$ Phase Transition in the Energetic Nitramine Octahydro-1,3,5,7-tetranitro-1,3,5,7-tetrazocine, J. Chem. Phys., 2004, 121(11), $5550-5552$.

[15] Li J., Brill T., Kinetics of Solid Polymorphic Phase Transitions of CL-20, Propellants Explos. Pyrotech., 2007, 32(4), 326-330.

[16] Flandrois S., Cinetique de Changements de Phase à l'etat Solide (Kinetics of Phase Transformations in Solid State), J. Chim. Phys., 1974, 71(6), 979-991.

[17] Nielsen A.T., Chafin A.P., Christian S.L., Moore D.W., Nadler M.P., Nissan R.A., Vanderah D.J., Synthesis of Polyazapolycyclic Caged Polynitramines, Tetrahedron, 1998, 54, 11793-11812.

[18] Chukanov N., Zakharov V., Chervonnyi A., Vozchikova S., Korsounskii B., Kinetics of Reversible Polymorphic Transitions in High-energy Compounds. Phase Transformations in Octahydro-1,3,5,7-tetranitro-1,3,5,7-tetrazocine, Russ. J. Phys. Chem. B, 2014, 8(5), 641-650.

[19] Latypov N.V., Johansson M., Holmgren E., Sizova E.V., Sizov V.V., Bellamy A.J., On the Synthesis of 1,1-Diamino-2,2-dinitroethene (FOX-7) by Nitration of 4,6-Dihydroxy-2-methylpyrimidine, Organic Process Research \& Development, 2007, 11(1), 56-59.

[20] Russell T., Miller P., Piermarini G., Pressure/Temperature Phase Diagram of Hexanitrohexaazaizowurzitene, J. Phys. Chem., 1993, 97(9), 1993-1997.

[21] Golovina N., Utenyshev A., Bozhenko K., Chukanov N., Zakharov V., Korsounskii B., The Energy Parameters of 2,4,6,8,10,12-Hexanitro-2,4,6,8,10,12hexaazaisowurtzitane Polymorphs and their Phase Transitions, Russ. J. Phys. Chem. A., 2009, 83(7), 1153-1159.

[22] Golovina N., Raevskii A., Chukanov N., Korsounskii B., Shilov G., Atovmyan L., Aldoshin S., Density of Crystals of 2,4,6,8,10,12-hexanitro-2,4,6,8,10,12hexaazaisowurzitane, a Potential Ligand (in Russian), Rossiyskiy Khimicheskiy 
Zhurnal, 2004, 48(1), 41-48.

[23] Bernstein J., Polymorphism in Molecular Crystals, Claredon Press, Oxford, 2002, p. 428; ISBN 0198506058.

[24] Taylor A., Atwood D., Physicochemical Principles of Pharmacy, Pharmaceutical Press, London, 2011, p. 510; ISBN 0853699844.

[25] Lempert D., Chukanov N., On the Use of Energetic Compounds Containing Small Molecules Occluded in the Structural Cavities of the Crystal, Combustion, Explosion, and Shock Waves (Engl. Transl.), 2014, 50(2), 238-241.

[26] Chukanov N., Golovina N., Nedelko V., Dubikhin V., Vozchikova S., Ananyina O., Larikova T., Nazin G., Korsounskii B., Aldoshin S., Volk F., Phase Transitions in Hexanitrohexaazaisowurtzitane, 32nd Int. Annu. Conf. ICT, Karlsruhe, Germany, 2001, 101/1-101/9.

[27] Chukanov N., Raevskii A., Golovina N., Aldoshin S., Korsounskii B., Nedelko V., Dubikhin V., Volk F., Kushnarenko I., On the Kinetics and Mechanism of Phase Transformations in Hexanitrohexaazaisowurtzitane. The Role of Water, Microstraining and Dislocations, 33th Аnnu. Conf. ICT, Karlsruhe, Germany, 2002, 105/1-105/12.

[28] Chukanov N., Korsounskii B., Nedelko V., Zakharov V., Golovina N., Raevskii A., Vozchikova S., Ananyina O., Larikova T., Aldoshin S., Kinetics of Dehydration and Phase Transitions in Hexanitrohexaazaisowurtzitane: Effect of Particle Sizes, 37th Int. Annu. Conf. ICT, Karlsruhe, Germany, 2006, 29/1-29/12.

[29] Chukanov N., Dubikhin V., Raevskii A., Golovina N., Korsounskii B., Nedelko V., Aldoshin S., Kinetics and Mechanism of Polymorphous Transition in Polycrystalline E-Hexanitrohexaazaisowurtzitane, Russ. J. Phys. Chem. A, 2006, 80(2), 281-287.

[30] Chukanov N., Zakharov V., Korsounskii B., Raevskii A., Nedelko V., Vozchikova S., Larikova T., Golovina N., Aldoshin S., The Kinetics of the Polymorphic Transition of the $\alpha$-form of 2,4,6,8,10,12-hexanitrohexaazaisowurtzitane, Russ. J. Phys. Chem. A, 2009, 83(1), 29-33.

[31] Chukanov N., Dubovitskii V., Zakharov V., Golovina N., Korsounskii B., Vozchikova S., Nedel'ko V., Larikova T., Raevskii A., Aldoshin S., Phase Transformations of 2,4,6,8,10,12-Hexanitrohexaazaisowurtzitane: the Role Played by Water, Dislocations, and Density, Russ. J. Phys. Chem. B, 2009, 3(3), 486-493.

[32] Crawford M.-J., Evers J., Göbel M., Klapötke T., Mayer P., Oehlinger G., Welch J., $\gamma$-FOX-7: Structure of a High Energy Density Material Immediately Prior to Decomposition, Propellants Explos. Pyrotech., 2007, 32(6), 478-495.

[33] Zakharov V., Korsunskii B., Kinetics of the $\beta \rightarrow \delta$ Structural Reorganization in 1,3,5,7-tetranitrooctahydro-1,3,5,7-tetrazocine, Russ. Chem. Bull., 2011, 60(7), 1436-1439.

[34] Zakharov V., Chukanov N., Chervonnyi A., Vozchikova S., Korsounskii B. Kinetics of Reversible Polymorphic Transition in Energetic Materials. Phase Transitions $\alpha \rightarrow \mathrm{B}$ and B $\rightarrow \alpha$ in 1,1-Diamino-2,2-dinitroethylene, Russ. J. Phys. Chem. B, 2014, 8(6), 822-828.

[35] Karpowicz R., Brill T., The $\beta \rightarrow \delta$ Transformation of HMX: Its Thermal Analysis 
and Relationship to Propellants, AIAA J., 1982, 20(11), 1586-1591.

[36] Saw C., Kinetics of HMX and Phase Transitions: Effects of Grain Size at Elevated Temperature, $12^{\text {th }}$ Symp. Int. Detonation, San Diego, California, USA, 2002, Published by Office of Naval Research, 2005, 70-76.

[37] Kempa P., Herrmann M., Metzger F., Thome V., Kjellström A., Latypov N., Phase Transitions of FOX-7 Studied by X-ray Diffraction and Thermal Analysis, $35^{\text {th }}$ Int. Annu. Conf. ICT, Karlsruhe, Germany, 2004, 71.1-71.15.

[38] Grigoriev D., Onthogeny of Minerals, Israel Program for Scientific Translations, Jerusalem, 1965, p. 250.

[39] Evers J., Klapötke T., Mayer P., Oehlinger G., Welch J., $\alpha$ - and $\beta$-FOX-7, Polymorphs of a High Energy Density Material, Studied by X-ray Single Crystal and Powder Investigations in the Temperature Range from 200 to $423 \mathrm{~K}$, Inorg. Chem., 2006, 45(13), 4996-5003.

[40] Main P., Cobbledick R., Small R., Structure of the Fourth Form of 1,3,5,7-tetranitro1,3,5,7-tetraazacyclooctane ( $\gamma$-HMX), $2 \mathrm{C}_{4} \mathrm{H}_{8} \mathrm{~N}_{8} \mathrm{O}_{8} \cdot 0.5 \mathrm{H}_{2} \mathrm{O}$, Acta Crystallogr., 1985, C41, 1351-1354.

[41] Deyun E., Chukanov N., Korsounskii B., A Kinetic Model of the Structural Transformation of a Molecular Crystal Initiated by the Escape of Mobile Defects, Russ. J. Phys. Chem., 2011, 5(1), 33-40. 